Supporting Information for:

\title{
Water Resistant and Flexible MOF Materials for Highly-Efficient Separation of Methane from Nitrogen
}

\author{
Xiao-Wei Liu, ${ }^{\dagger}, \S$ Yi-Ming Gu ${ }^{\dagger, \S}$ Tian-Jun Sun, ${ }^{* \dagger}$ Ya Guo, ${ }^{\dagger, \S}$ Xiao-Li Wei, ${ }^{\dagger,}$ Sheng-Sheng \\ Zhao $^{\dagger}$ and Shu-Dong Wang* ${ }^{\dagger}$ \\ 'Dalian National Laboratory for Clean Energy (DNL), Dalian Institute of Chemical Physics (DICP), \\ Chinese Academy of Sciences (CAS), 457 Zhongshan Road, Dalian 116023, P. R. China \\ ${ }^{\S}$ University of Chinese Academy of Sciences (UCAS), 19 A Yuquan Road, Beijing 100049, P. R. China \\ Email:wangsd@dicp.ac.cn, suntianjun@dlmu.edu.cn
}

\section{Contents:}

\section{S1. Supporting Figures and Tables}

\section{S2. References}




\section{Supporting Figures and Tables}

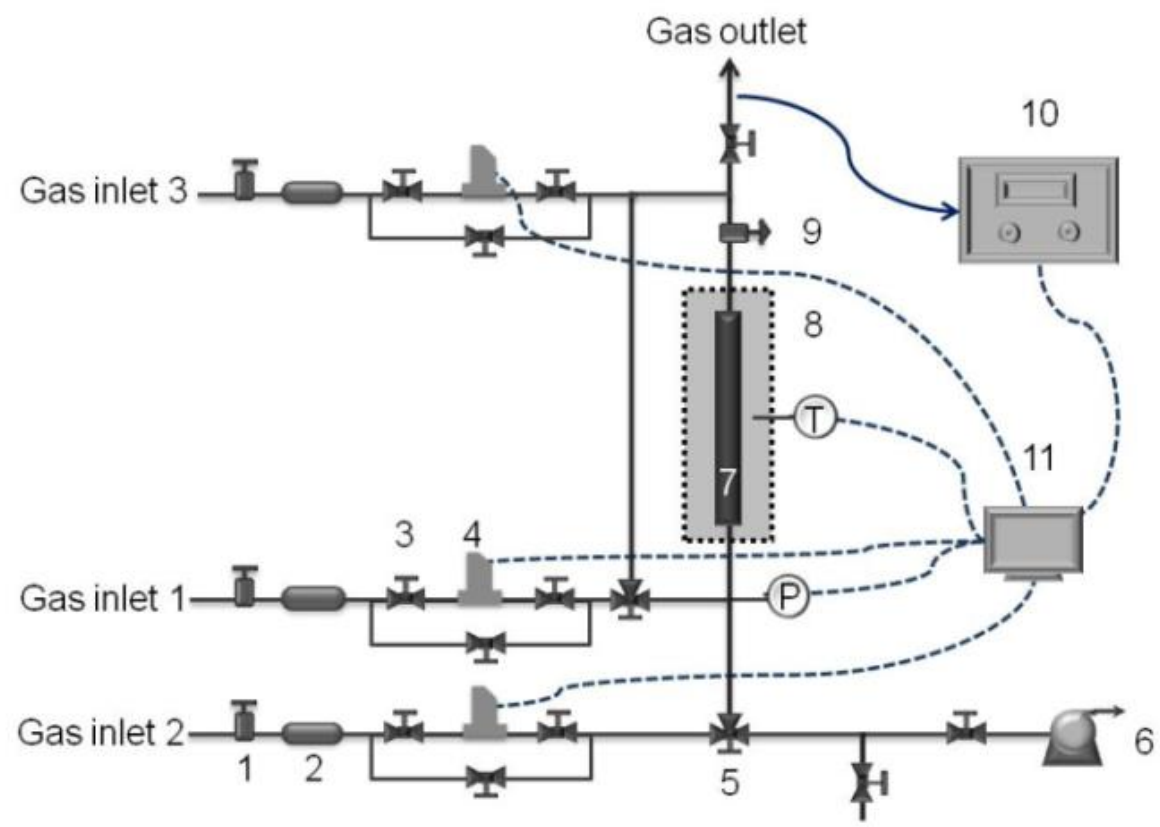

Figure S1. Schematic of the set-up for breakthrough experiments. 1. pressure reducing regulator; 2. gas purifier; 3 . two-way valve; 4. mass flowmeter; 5 . three-way valve; 6 . vacuum pump; 7. adsorbent fixed-bed; 8. thermostatic chamber; 9 . back pressure regulator; 10. mass spectrometer; 11. computer.
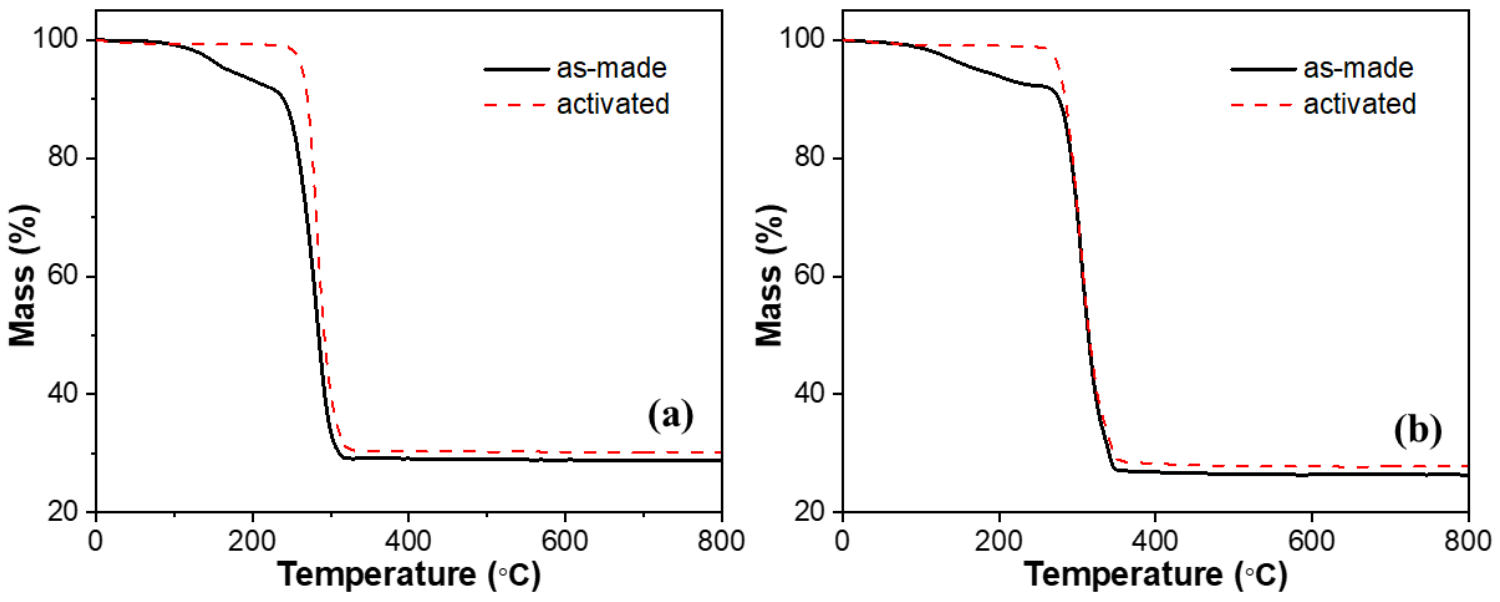

Figure S2. TGA patterns of (a) Co-MA-BPY and (b) Ni-MA-BPY MOFs: the as-made (black solid line) and the guest-free (red dash line) samples. 


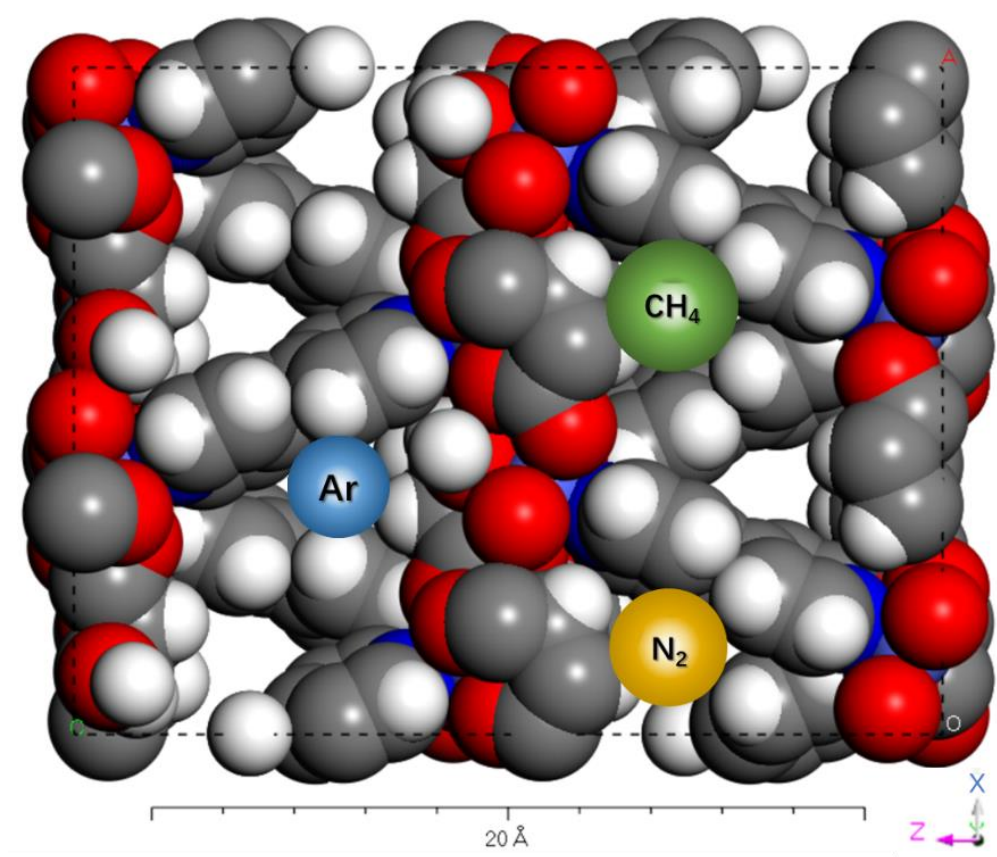

PLD of Co-MA-BPY: $2.5 \AA$

PLD of Ni-MA-BPY: $3.0 \AA$

Kinetic diameter of $\operatorname{Ar}(3.4 \AA)$, $\mathrm{CH}_{4}(3.8 \AA)$ and $\mathrm{N}_{2}(3.6 \AA)$

Figure S3. Schematic showing larger gas molecules inaccessible to the pore channels of M-MABPY MOFs of the original state. ${ }^{1}$ Key of atoms: Co or Ni (pale blue), $\mathrm{C}$ (grey), O (red), N (blue) and $\mathrm{H}$ (white).

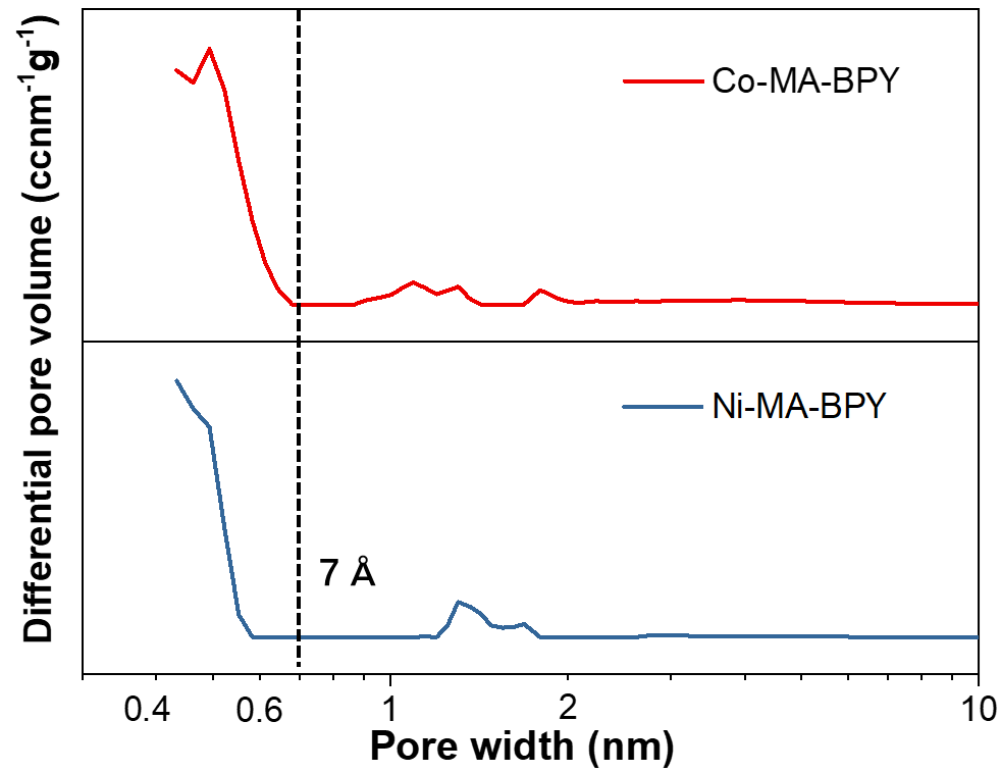

Figure S4. Pore size distributions (PSDs) of Co-MA-BPY (red solid line) and Ni-MA-BPY (blue dash line) MOFs. PSDs are calculated by using a NLDFT cylindrical pores model. 

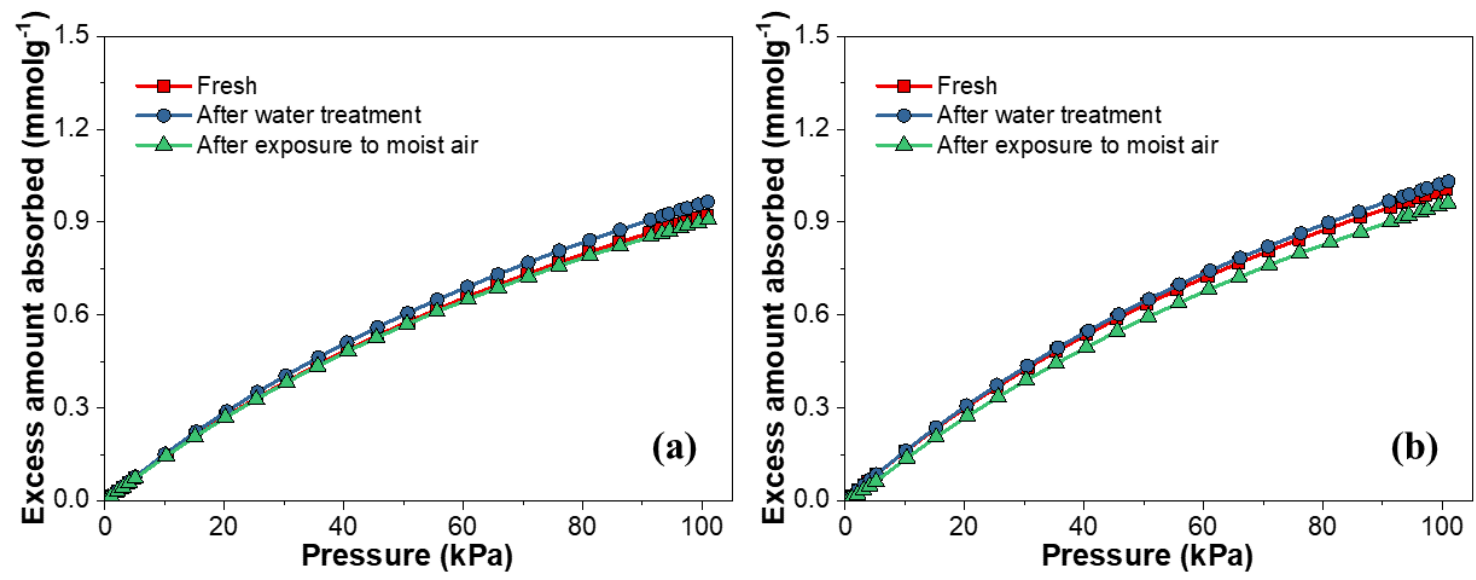

Figure S5. Pure $\mathrm{CH}_{4}$ adsorption isotherms of different (a) Co-MA-BPY and (b) Ni-MA-BPY MOF samples at $298 \mathrm{~K}$ : fresh (red squares), after being soaked in water for $24 \mathrm{~h}$ (blue circles) and after being exposed to $75 \% \mathrm{RH}$ air atmosphere for 2 weeks (green triangles).
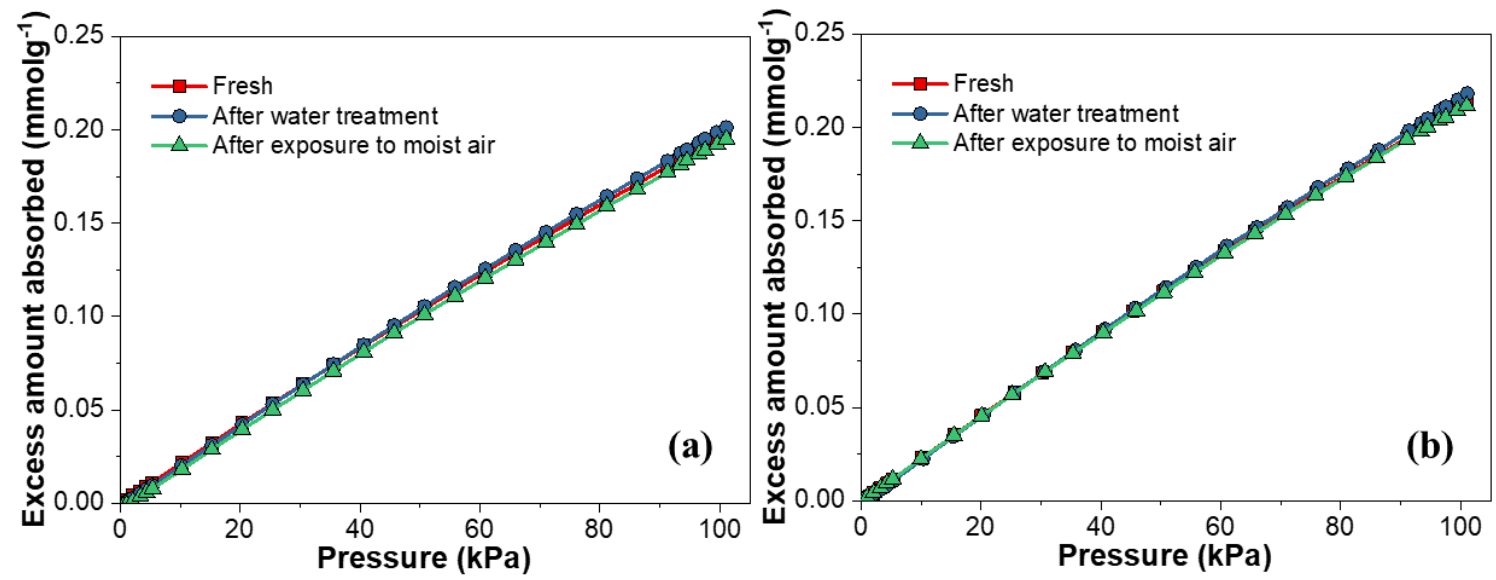

Figure S6. Pure $\mathrm{N}_{2}$ adsorption isotherms of different (a) Co-MA-BPY and (b) Ni-MA-BPY MOF samples at $298 \mathrm{~K}$ : fresh (red squares), after being soaked in water for $24 \mathrm{~h}$ (blue circles) and after being exposed to $75 \% \mathrm{RH}$ air atmosphere for 2 weeks (green triangles). 

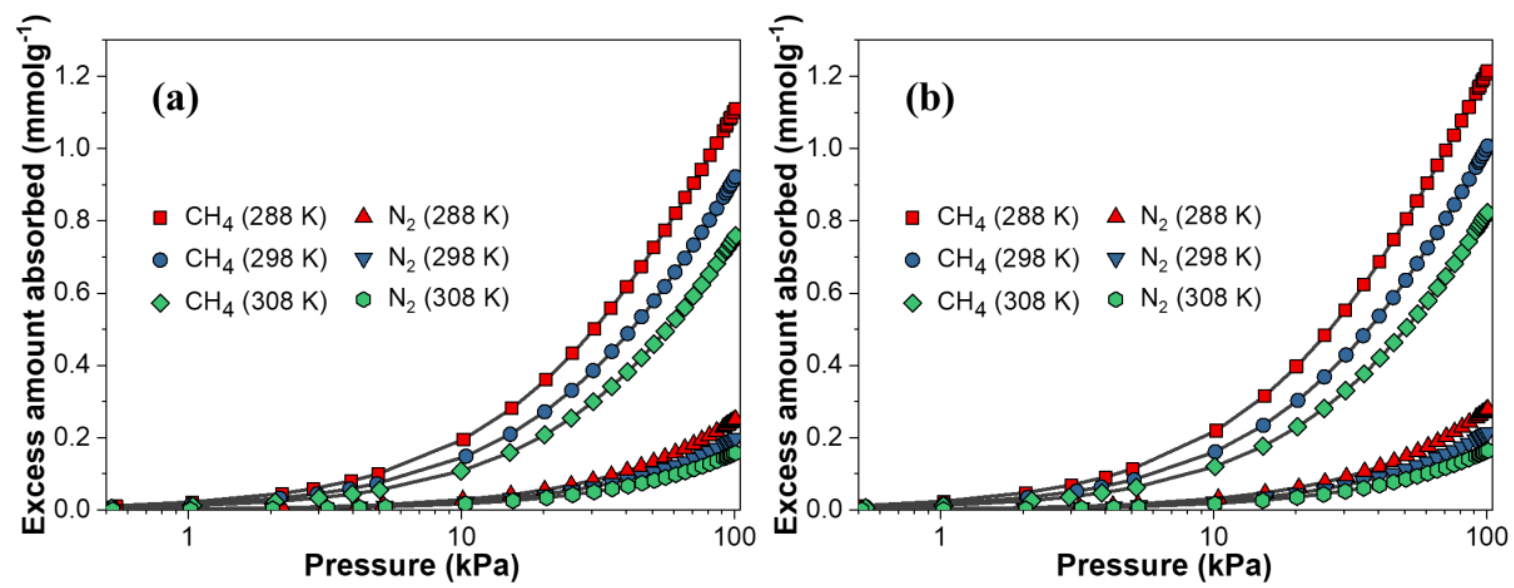

Figure S7. Pure $\mathrm{CH}_{4}$ (squares, circles and diamonds) and $\mathrm{N}_{2}$ (triangles and hexagons) adsorption isotherms of (a) Co-MA-BPY and (b) Ni-MA-BPY MOF samples at 288 (red), 298 (blue) and $308 \mathrm{~K}$ (green) based on a X-logarithmic scale. Black lines indicate the dual sites LangmuirFreundlich fitting data. 

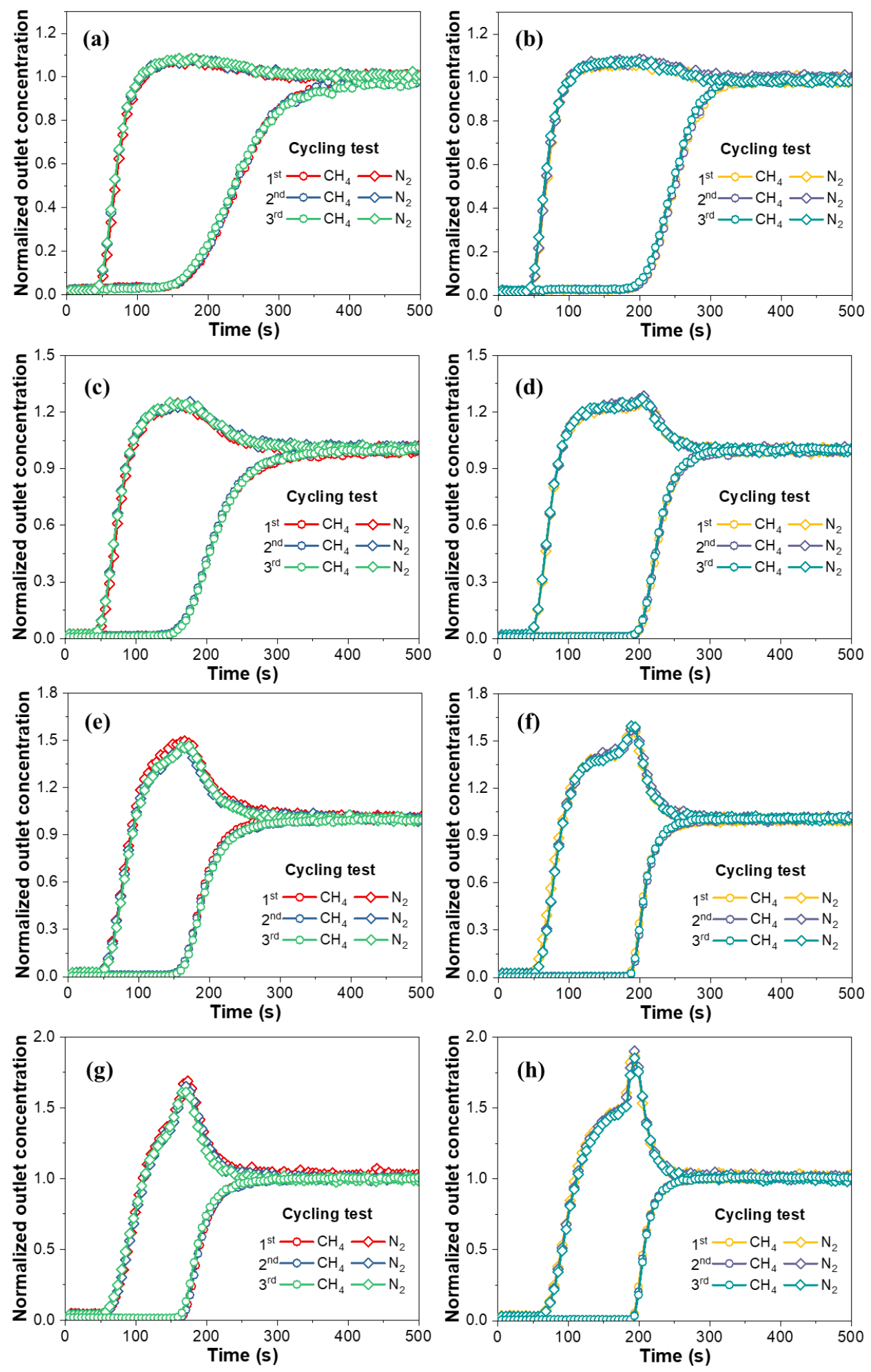

Figure S8. Experimental column breakthrough curves of cycling tests for $\mathrm{CH}_{4} / \mathrm{N}_{2}$ binary mixtures with different compositions (v/v) (a, b) 5: 95, (b, d) 15: 85, (e, f) 30: 70 and (g, h) 50:50 in an absorber bed packed with Co-MA-BPY (a, c, e and $\mathbf{g})$ and Ni-MA-BPY (b, d, f and h) at $298 \mathrm{~K}$ and 1.0 bar. 
Table S1.Textual properties of the porous structures of M-MA-BPY ( $\mathrm{M}=\mathrm{Co}$ and Ni) MOFs characterized using Zeo++ software package based on the reported structures ${ }^{1}$ and calculated from their experimental argon adsorption isotherms at $87 \mathrm{~K}$.

\begin{tabular}{ccccccc}
\hline Adsorbents & $\begin{array}{c}L C D^{\mathrm{a}} \\
(\AA)\end{array}$ & $\begin{array}{c}P L D^{\mathrm{a}} \\
(\AA)\end{array}$ & $\begin{array}{c}S_{B E T} \\
\left(\mathrm{~m}^{2} \cdot \mathrm{g}^{-1}\right)\end{array}$ & $\begin{array}{c}S_{\text {Langmuir }} \\
\left(\mathrm{m}^{2} \cdot \mathrm{g}^{-1}\right)\end{array}$ & $\begin{array}{c}V_{t}^{\mathrm{b}} \\
\left(\mathrm{cm}^{3} \cdot \mathrm{g}^{-1}\right)\end{array}$ & $\begin{array}{c}V_{\text {mic }}{ }^{\mathrm{c}} \\
\left(\mathrm{cm}^{3} \cdot \mathrm{g}^{-1}\right)\end{array}$ \\
\hline Co-MA-BPY & 5.223 & 2.520 & 451 & 575 & 0.230 & 0.142 \\
Ni-MA-BPY & 5.098 & 3.022 & 464 & 544 & 0.191 & 0.160 \\
\hline
\end{tabular}

${ }^{\mathrm{a}} \mathrm{LCD}$ and PLD were characterized using the open-source Zeo++ software package, ${ }^{\mathrm{b}} V_{t}$ (total pore volume) was calculated by Gurvich-rule at $\mathrm{P} / \mathrm{P}_{0}=0.95,{ }^{\mathrm{c}} V_{\text {mic }}$ (micropore volume) was calculated by t-Plot method. Here micropore suggests the pores with a diameter smaller than $2.0 \mathrm{~nm}$. 
Table S2. Fitting parameters of the DSLF model for $\mathrm{CH}_{4}$ and $\mathrm{N}_{2}$ adsorption on Co-MA-BPY MOF at different temperature

\begin{tabular}{lllllll}
\hline Adsorbates & $\mathrm{CH}_{4}$ & $\mathrm{~N}_{2}$ & $\mathrm{CH}_{4}$ & $\mathrm{~N}_{2}$ & $\mathrm{CH}_{4}$ & $\mathrm{~N}_{2}$ \\
\cline { 2 - 7 } $\mathrm{T} / \mathrm{K}$ & 288 & 288 & 298 & 298 & 308 & 308 \\
\hline $\mathrm{q}_{1}\left(\mathrm{mmol} \cdot \mathrm{g}^{-1}\right)$ & 2.2543 & 1.5286 & 2.1688 & 1.5283 & 2.0377 & 1.5865 \\
$\mathrm{~b}_{1}\left(\mathrm{kPa}^{-1}\right)$ & $9.1332 \mathrm{E}-03$ & $1.3797 \mathrm{E}-03$ & $6.7964 \mathrm{E}-03$ & $1.0395 \mathrm{E}-03$ & $5.1956 \mathrm{E}-03$ & $7.3382 \mathrm{E}-04$ \\
$\mathrm{c}_{1}$ & 0.9937 & 0.9919 & 0.9995 & 0.9848 & 1.0095 & 1.0056 \\
$\mathrm{q}_{2}\left(\mathrm{mmol}^{-1} \mathrm{~g}^{-1}\right)$ & 0.8882 & 0.8883 & 0.8715 & 0.8524 & 0.8424 & 0.7656 \\
$\mathrm{~b}_{2}\left(\mathrm{kPa}^{-1}\right)$ & $8.9575 \mathrm{E}-04$ & $8.8943 \mathrm{E}-04$ & $8.8119 \mathrm{E}-04$ & $8.6464 \mathrm{E}-04$ & $8.5628 \mathrm{E}-04$ & $7.9892 \mathrm{E}-04$ \\
$\mathrm{c}_{2}$ & 0.8822 & 0.9889 & 0.8696 & 0.9738 & 0.8635 & 0.9459 \\
\hline
\end{tabular}

Table S3. Fitting parameters of the DSLF model for $\mathrm{CH}_{4}$ and $\mathrm{N}_{2}$ adsorption on Ni-MA-BPY MOF at different temperature

\begin{tabular}{lllllll}
\hline Adsorbates & $\mathrm{CH}_{4}$ & $\mathrm{~N}_{2}$ & $\mathrm{CH}_{4}$ & $\mathrm{~N}_{2}$ & $\mathrm{CH}_{4}$ & $\mathrm{~N}_{2}$ \\
\cline { 2 - 7 } $\mathrm{T} / \mathrm{K}$ & 288 & 288 & 298 & 298 & 308 & 308 \\
\hline $\mathrm{q}_{1}\left(\mathrm{mmol} \cdot \mathrm{g}^{-1}\right)$ & 2.3736 & 1.9444 & 2.2537 & 1.4343 & 2.1201 & 1.2401 \\
$\mathrm{~b}_{1}\left(\mathrm{kPa}^{-1}\right)$ & $9.6882 \mathrm{E}-03$ & $1.2335 \mathrm{E}-03$ & $7.1805 \mathrm{E}-03$ & $1.1617 \mathrm{E}-03$ & $5.4622 \mathrm{E}-03$ & $9.7995 \mathrm{E}-04$ \\
$\mathrm{c}_{1}$ & 0.9979 & 0.9929 & 1.0069 & 0.9997 & 1.0152 & 0.9957 \\
$\mathrm{q}_{2}\left(\mathrm{mmol} \cdot \mathrm{g}^{-1}\right)$ & 0.9059 & 0.8936 & 0.8764 & 0.8637 & 0.8592 & 0.8092 \\
$\mathrm{~b}_{2}\left(\mathrm{kPa}^{-1}\right)$ & $8.8772 \mathrm{E}-04$ & $8.9472 \mathrm{E}-04$ & $8.8462 \mathrm{E}-04$ & $8.6710 \mathrm{E}-04$ & $8.7192 \mathrm{E}-04$ & $8.5242 \mathrm{E}-04$ \\
$\mathrm{c}_{2}$ & 0.8975 & 0.9858 & 0.8799 & 0.9774 & 0.8593 & 0.9577 \\
\hline
\end{tabular}


Table S4. Selectivities of $\mathrm{CH}_{4} / \mathrm{N}_{2}$ and the $\mathrm{CH}_{4}$ sorption capacity of some selected adsorbents at $298 \mathrm{~K}$ and 1 bar.

\begin{tabular}{llll}
\hline Adsorbents & $\begin{array}{l}\text { Selectivity } \\
\left(\mathrm{CH}_{4} / \mathrm{N}_{2} 50: 50, \mathrm{v} / \mathrm{v}\right)\end{array}$ & $\begin{array}{l}\mathrm{CH}_{4} \text { Capacity } \\
\left(\mathrm{mmol} \cdot \mathrm{g}^{-1}\right)\end{array}$ & Ref. \\
\hline Co-MA-BPY & 7.16 & 0.92 & This work \\
Ni-MA-BPY & 7.41 & 1.01 & This work \\
Zeolite-5A & 0.94 & 0.81 & 2 \\
MOF-5 & 1.13 & 0.13 & 2 \\
$\mathrm{Cu}($ me-4py-trz-ia $)$ & 4.20 & 1.12 & 3 \\
$\mathrm{ATC}-\mathrm{Cu}$ & 9.70 & 2.90 & 4 \\
$\mathrm{HKUST}-1$ & 3.70 & 0.90 & 4 \\
$\mathrm{Ni}(\mathrm{HCOO})_{6}$ & 6.20 & 0.78 & 5 \\
$\mathrm{Co}(\mathrm{HCOO})_{6}$ & 5.50 & 0.79 & 6 \\
$\mathrm{Cu}(\mathrm{INA})_{2}$ & 6.90 & 0.83 & 7 \\
$\mathrm{Co}_{3}\left(\mathrm{C}_{4} \mathrm{O}_{4}\right)(\mathrm{OH})_{2}$ & 12.50 & 0.40 & 8 \\
$\mathrm{USTA}-30 \mathrm{a}$ & 5.00 & 0.63 & 9 \\
$\mathrm{Cu}-\mathrm{MOF}$ & 6.90 & 0.47 & 10 \\
$\mathrm{Ni}-\mathrm{MOF}-74$ & 3.80 & 1.91 & 11 \\
$\mathrm{Co}-\mathrm{MOF}-74$ & 3.20 & 1.63 & 11 \\
$\mathrm{Mg}-\mathrm{MOF}-74$ & 1.50 & 1.66 & \\
\hline
\end{tabular}




\section{Reference}

1. Duan, L.-M.; Xie, F.-T.; Chen, X.-Y.; Chen, Y.; Lu, Y.-K.; Cheng, P.; Xu, J.-Q., Syntheses, structures, and magnetic properties of three novel metal-malate-bipyridine coordination polymers with layered and pillared topology. Cryst. Growth Des. 2006, 6 (5), 1101-1106.

2. Saha, D.; Bao, Z.; Jia, F.; Deng, S., Adsorption of $\mathrm{CO}_{2}, \mathrm{CH}_{4}, \mathrm{~N}_{2} \mathrm{O}$, and $\mathrm{N}_{2}$ on MOF-5, MOF177, and Zeolite 5A. Environ. Sci. Technol. 2010, 44 (5), 1820-1826.

3. Möllmer, J.; Lange, M.; Möller, A.; Patzschke, C.; Stein, K.; Lässig, D.; Lincke, J.; Gläser, R.; Krautscheid, H.; Staudt, R., Pure and mixed gas adsorption of $\mathrm{CH}_{4}$ and $\mathrm{N}_{2}$ on the metalorganic framework Basolite®A100 and a novel copper-based 1,2,4-triazolyl isophthalate MOF. J. Mater. Chem. 2012, 22 (20), 10274-10286.

4. Niu, Z.; Cui, X.; Pham, T.; Lan, P. C.; Xing, H.; Forrest, K. A.; Wojtas, L.; Space, B.; Ma, S., A metal-organic framework based methane nano-trap for the capture of coal-mine methane. Angew. Chem. Int. Ed. 2019, https://doi.org/10.1002/anie.201904507.

5. Liu, X.-W.; Guo, Y.; Tao, A.; Fischer, M.; Sun, T.-J.; Moghadam, P. Z.; Fairen-Jimenez, D.; Wang, S.-D., "Explosive" synthesis of metal-formate frameworks for methane capture: an experimental and computational study. Chem. Commun. 2017, 53 (83), 11437-11440.

6. Hu, J.; Sun, T.; Liu, X.; Zhao, S.; Wang, S., Rationally tuning the separation performances of $\left[\mathrm{M}_{3}(\mathrm{HCOO})_{6}\right]$ frameworks for $\mathrm{CH}_{4} / \mathrm{N}_{2}$ mixtures via metal substitution. Micropor. Mesopor. Mater. 2016, 225, 456-464.

7. Hu, J.; Sun, T.; Liu, X.; Guo, Y.; Wang, S., Separation of $\mathrm{CH}_{4} / \mathrm{N}_{2}$ mixtures in metal-organic frameworks with 1D micro-channels. RSC Adv. 2016, 6 (68), 64039-64046.

8. Liangying, L.; Lifeng, Y.; Jiawei, W.; Zhiguo, Z.; Qiwei, Y.; Yiwen, Y.; Qilong, R.; Zongbi, B., Highly efficient separation of methane from nitrogen on a squarate-based metal-organic framework. AlChE J. 2018, 64, 3681-3689.

9. He, Y.; Xiang, S.; Zhang, Z.; Xiong, S.; Fronczek, F. R.; Krishna, R.; O'Keeffe, M.; Chen, B., A microporous lanthanide-tricarboxylate framework with the potential for purification of natural gas. Chem. Commun. 2012, 48 (88), 10856-10858.

10. Wu, X.; Yuan, B.; Bao, Z.; Deng, S., Adsorption of carbon dioxide, methane and nitrogen on an ultramicroporous copper metal-organic framework. J. Colloid Interf. Sci. 2014, 430, 78-84.

11. Li, L.; Yang, J.; Li, J.; Chen, Y.; Li, J., Separation of $\mathrm{CO}_{2} / \mathrm{CH}_{4}$ and $\mathrm{CH}_{4} / \mathrm{N}_{2}$ mixtures by M/DOBDC: A detailed dynamic comparison with MIL-100(Cr) and activated carbon. Micropor. Mesopor. Mater. 2014, 198, 236-246. 